\title{
In vitro evaluation of neuraminidase inhibitors using the neuraminidase-dependent release assay of hemagglutinin-pseudotyped viruses
}

\author{
Ching-Yao Su ${ }^{\mathrm{a}, \mathrm{b}}$, Shi-Yun Wang ${ }^{\mathrm{a}}$, Jiun-Jie Shie ${ }^{\mathrm{a}}$, King-Song Jeng ${ }^{\mathrm{c}}$, Nigel J. Temperton ${ }^{\mathrm{d}}$, \\ Jim-Min Fang ${ }^{\mathrm{a}, \mathrm{e}}$, Chi-Huey Wong ${ }^{\mathrm{a}, \mathrm{b}}$, Yih-Shyun E. Cheng ${ }^{\mathrm{a}, *}$ \\ ${ }^{a}$ Genomics Research Center, Academia Sinica, 128 Academia Road, Section 2, Taipei 115, Taiwan, ROC \\ ${ }^{\mathrm{b}}$ Institute of Biochemical Sciences, National Taiwan University, 1 Roosevelt Road, Section 4, Taipei 106, Taiwan, ROC \\ ' Institute of Molecular Biology, Academia Sinica, 128 Academia Road, Section 2, Taipei 115, Taiwan, ROC \\ ${ }^{\mathrm{d}}$ MRC/UCL Centre for Medical Molecular Virology, Division of Infection and Immunity, University College London, \\ 46 Cleveland Street, London W1T4JF, United Kingdom \\ e Department of Chemistry, National Taiwan University, 1 Roosevelt Road, Section 4, Taipei 106, Taiwan, ROC
}

\section{A R T I C L E I N F O}

\section{Article history:}

Received 29 October 2007

Accepted 17 March 2008

\section{Keywords:}

Neuraminidase inhibitor

Pseudotype virus

Antiviral

Viral release assay

Drug resistance

\begin{abstract}
A B S T R A C T
For the treatment of influenza virus infections, neuraminidase inhibitors (NAIs) that prevent the release of virus particles have been effective against most influenza strains. Several neuraminidase (NA) assays are available for the evaluation of NAIs. To understand the NAI functions under physiological conditions, assays mimicking viral particle release should be useful. We have constructed retrovirus-based reporter viruses that are pseudotyped with hemagglutinin (HA) glycoprotein by transfection of producer cells using plasmids expressing retroviral gag-pol, influenza HA, NA, and firefly luciferase genes. Similarly to the life cycle of influenza viruses, the release of pseudotype viruses also requires neuraminidase functions. This requirement was used to develop an assay to evaluate NAI activities by measuring inhibition of pseudotype virus production at different NAI concentrations. The pseudotype virus release assay was used to determine the $\mathrm{IC}_{50}$ values of Oseltamivir carboxylate, Zanamivir, and the novel phosphonate congeners of Oseltamivir against $\mathrm{N} 1$ group neuraminidases and their $\mathrm{H} 274 \mathrm{Y}$ Oseltamivir carboxylate-resistant mutants. The deduced $\mathrm{IC}_{50}$ values obtained using the release assay correlated with those determined using the fluorogenic substrate 2 '-(4-methylumbelliferyl)- $\alpha$-D- $N$-acetylneuraminic acid (MUNANA) and also correlated with the infectivity results.
\end{abstract}

(c) 2008 Elsevier B.V. All rights reserved.

\section{Introduction}

Influenza virus infects avian and many species of mammals. Seasonal human infections by influenza viruses result in mortality and financial losses and are managed predominantly by vaccination (Chowell et al., 2007; Vila-Córcoles et al., 2007). The recent H5N1 avian influenza viruses that are highly pathogenic to several wild and domestic avian species infected more than 300 persons, many fatally, in many Asian countries and raised the concern of another influenza pandemic (Hampson, 2006; Poland et al., 2007). Among the therapeutic options for the treatment and prevention of influenza infections, neuraminidase inhibitors (NAI) are most promising (Alymova et al., 2005; De Clercq, 2006). Before an effective vaccine for avian influenza is developed, NAIs are crucial

\footnotetext{
* Corresponding author. Tel.: +88622789 9930x334; fax: +886227899931.

E-mail address: ysecheng@gate.sinica.edu.tw (Y.-S.E. Cheng).
}

ammunition for protection against the possible pandemic H5N1 viruses that are often resistant to the other class of approved antiflu agents: adamantanamine derivatives (Gani et al., 2005; Hurt et al., 2007).

Several neuraminidase assays are currently used for the evaluation of NAIs. The substrates of these assays are usually synthetic sialic acid derivatives that upon cleavage by neuraminidase produce convenient reporting signals for the measurements (Buxton et al., 2000; Cabezas et al., 1983; França de Barros et al., 2003; Onodera, 1994). In order to understand NAI inhibition under physiological conditions, cell-based assays are helpful as the neuraminidase reaction does not simply involve the terminal sialic acid of the substrates. The physiological functions of influenza neuraminidase are thought to enhance the spread of the influenza infection in several ways. After replication, packaging, and budding of progeny viruses, the neuraminidases are required to cleave the cell surface sialic acids to release the progeny virus from infected cells (Palese et al., 1974). The neuraminidase also prevents the aggregation of 
the released influenza viruses for efficient infections (Bhatia and Kast, 2007; Palese et al., 1974). In addition, the viral associated neuraminidase is thought to cleave the sialic acid of mucins in the respiratory tract thus facilitating access to epithelial target cells for productive infections (Bhatia and Kast, 2007). Therefore, the natural substrates of influenza neuraminidase are terminal sialic acids located at the producer cells, the viral surface, and various animal tissues. Furthermore, the carbohydrate structures adjacent to the terminal sialic acid were reported to be crucial to the specificity and reactivity of different influenza neuraminidases (Katinger et al., 2004; Kiyotani et al., 1987; Kobasa et al., 1999; Sato et al., 1998). The properties of the natural substrates are difficult to be included in an enzymatic assay using a single synthetic substrate. In addition, the influenza HA (hemagglutinin) that interacts with a variety of sialyl oligosaccharides also affects the catalysis of neuraminidases. This was illustrated by the reported NAI resistant mutations mapped to HA (Bantia et al., 1998; Cheam et al., 2004; Mishin et al., 2005; Molla et al., 2002). Therefore, a cell-based neuraminidase assay with the flexibility to measure inhibitor potencies against various neuraminidase and hemagglutinin mutations in different cells could contribute to the understanding of NAI activities under physiological conditions mimicking the viral release processes.

In the course of our study for the preparation of MuLV (murine leukemia virus) reporter virus pseudotyped with influenza HA glycoprotein, we established the requirement of neuraminidase activity for the production of pseudotyped viruses. This requirement was used to develop a cell-based assay, which closely mimics the influenza release process, for the evaluation of neuraminidase activities. This assay was used to determine the activities of several neuraminidase inhibitors against both wild type and mutant neuraminidases.

\section{Materials and methods}

\subsection{Viruses, cell lines, antibodies, compounds, and reagents}

Influenza A/WSN/1933 (H1N1) (from Dr. Shin-Ru Shih, Chang Gung University, Taiwan) was cultured in the allantoic cavities of 10-day-old embryonated chicken eggs for $72 \mathrm{~h}$, and purified by sucrose gradient centrifugation. Madin-Darby canine kidney (MDCK), and 293T cells were obtained from American Type Culture Collection (Manassas, VA), and were grown in DMEM (Dulbecco's modified Eagle's medium) containing 10\% fetal bovine serum and penicillin-streptomycin at $37^{\circ} \mathrm{C}$ under $5 \% \mathrm{CO}_{2}$. A549 cells also obtained from American Type Culture Collection, were cultured in RPMI 1640 (GibcoBRL, Gaithersburg, MD) containing 10\% fetal bovine serum and penicillin-streptomycin at $37^{\circ} \mathrm{C}$ under $5 \% \mathrm{CO}_{2}$. The antibodies used in this study were anti-HA (from LTK Biolaboratories, Taipei, Taiwan), anti-NA antibody (from Abcam, Taipei, Taiwan), antibody for p30 (produced from hybridoma cells, R187, ATCC, Manassas, VA), and anti-beta-actin antibody (from Sigma, St. Louis, MO). Oseltamivir carboxylate, Zanamivir and two phosphonate analogs of Oseltamivir (Compounds 3 and $\mathbf{4}$ ) were synthesized as described (Shie et al., 2007). Britelite assay kit for luciferase assays was purchased from PerkinElmer (Waltham, MA). All other reagents used in this work were purchased from Sigma (St. Louis, MO).

\subsection{Plasmids used and constructions}

Plasmids encoding HA [A/Viet Nam/1194/2004, GenBank EF541402 (Li et al., 2004)] and NA proteins [A/WSN/1933 TS61, GenBank CY010790] were subcloned into pcDNA3.1 (Invitrogen, Carlsbad, CA). The plasmids for the MuLV retrovirus expression system, pGagPol and pMuLV-Fluc (for reporter firefly luciferase expression) were reported previously (Temperton et al., 2007). The NA gene was mutated to introduce the H274Y mutation by the GeneTailor Site-Directed Mutagenesis System (Invitrogen) according to the manufacturer's instructions. Both the wild type and the mutant NA genes were cloned into pcDNA6 (Invitrogen). Plasmid DNA of the NA genes were used to transfect 293T cells to express recombinant neuraminidase at the cell surface. The transfected cells were collected at $48 \mathrm{~h}$, washed twice with PBS and used for the evaluation of neuraminidase inhibitors.

\subsection{Pseudotyped virus preparation, and purification}

Human 293T cells in 15-cm dishes were transfected with $4 \mu \mathrm{g}$ of pGag/Pol(MuLV), $6 \mu \mathrm{g}$ of pCNCL(MLV-Fluc), $2 \mu \mathrm{g}$ of pVSV or $4 \mu \mathrm{g}$ of pHA (from A/Vietnam/1194/2004 (H5N1)) and $3 \mu \mathrm{g}$ of pNA (from A/WSN/1933 (H1N1) supplemented with $51 \mu$ l lipofectamine 2000 (Invitrogen, Carlsbad, CA), and replenished with fresh media after overnight incubation. The HA-pseudotyped MuLV (HA-PV) preparations were harvested from the conditioned media, filtered through $0.45 \mu \mathrm{m}$ filters, and either used immediately or frozen at $-80^{\circ} \mathrm{C}$. HA-PV was purified by centrifugation for $2 \mathrm{~h}$ at $25,000 \mathrm{rpm}$ in a Beckman SW29 rotor over a $20 \%$ sucrose cushion.

\subsection{Antigen determinations}

The hemagglutinin contents were determined by ELISA according to the manufacturer's instructions (Bio Assay Systems, Hayward, CA). Immunoblot analyses of hemagglutinin after SDSpolyacrylamide gel electrophoresis were performed using a rabbit polyclonal antibody raised against a synthetic peptide (ASSGVSSACPYLGKSSFFRN). Other antibodies used for immunoblot studies were monoclonal antibody AC-15 for $\beta$-actin (Sigma, St. Louis, MO), and rabbit antibody for influenza neuraminidase (ab21305) from $A b$ Cam (Gene Research Lab Co., Taipei, Taiwan).

\subsection{Determination of pseudotyped virus titer by transduction}

Ten thousand A549 cells were plated onto the wells of 96-well plates 1 day prior to transduction and incubated with $100 \mu \mathrm{l}$ pseudotyped virus for $2 \mathrm{~h}$. After washing, cells were supplemented with fresh media, and incubated for an additional $48 \mathrm{~h}$. The luciferase activity was measured using the Britelite assay kit (PerkinElmer, Waltham, MA) and used according to the manufacturer's instructions.

\subsection{Pseudotyped virus release assay for NAI evaluations}

293T cells were plated onto 10 -cm dishes 1 day prior to transfection. Cells were transfected with $2 \mu \mathrm{g}$ pGagPol, $3 \mu \mathrm{g}$ pFluc, $2 \mu \mathrm{g}$ pHA, and $2 \mu \mathrm{g}$ pNA. Cells were recovered and re-plated in 96-well plates after $24 \mathrm{~h}$ and treated with NAI at various concentrations. After $48 \mathrm{~h}$, pseudotype viruses were collected by filtration through a 96-well filtration system (Millipore, Billerica, MA). The titers of the released pseudotype virus were determined by the expressed luciferase activities of transduced 293T cells using the Britelite assay kit as described in Section 2.5.

\subsection{Other assays}

Neuraminidase activity was measured in $0.33 \mathrm{M}$ MES, $4 \mathrm{mM}$ $\mathrm{CaCl}_{2}, 2.5 \%$ dimethyl sulfoxide, $\mathrm{pH} 6.5$ using a fluorogenic substrate, at $200 \mu \mathrm{M}$, of $2^{\prime}$-(4-methylumbelliferyl)- $\alpha$-D- $N$-acetylneuraminic acid (MUNANA; Sigma). The reaction was stopped with stop 

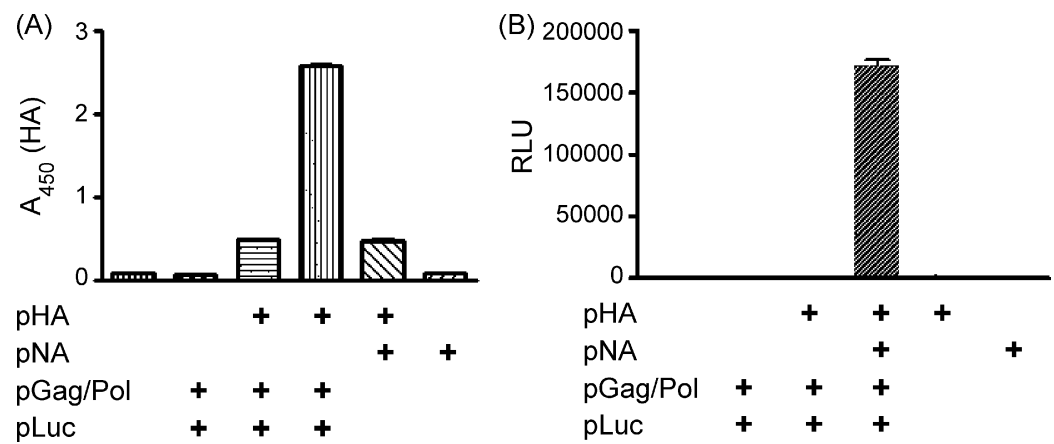

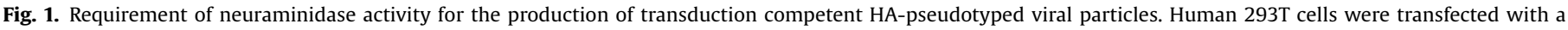

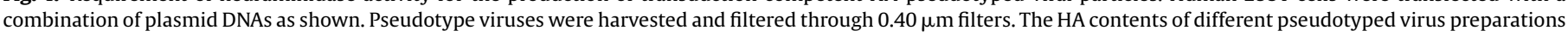
were measured by ELISA (A), and their activities to transduce A549 cells for luciferase expression are shown (B).

buffer $(0.5 \mathrm{M} \mathrm{NaOH}, 25 \%$ ethyl alcohol) after 15 min incubation at room temperature. The fluorescence of the released 4-methylumbelliferone was measured in an Envision plate reader (PerkinElmer, Wellesley, MA) using excitation and emission wavelengths of 365 and $460 \mathrm{~nm}$, respectively (Cabezas et al., 1983). $\mathrm{IC}_{50}$ determination was performed by fitting the curve of percent control activity without NAI versus NAI concentrations using Graph Pad Prism 4.

The anti-influenza activities of neuraminidase inhibitors were measured as $\mathrm{IC}_{50}$ values corresponding to the concentration of NA inhibitors required for $50 \%$ protection from $\mathrm{H} 1 \mathrm{~N} 1$-mediated cytopathic effects. Fifty $\mu$ l diluted $\mathrm{H} 1 \mathrm{~N} 1$ at $100 \mathrm{CCID}_{50}(50 \%$ cell culture infective dose) were mixed with equal volumes of NAI at various concentrations, and the mixtures were used to infect $1 \times 10^{5} \mathrm{MDCK}$ cells in 96-wells. After $48 \mathrm{~h}$ incubation at $37{ }^{\circ} \mathrm{C}$ and $5.0 \% \mathrm{CO}_{2}$, the cytopathic effects were determined with the CellTiter $96^{\circledR}$ AQueous Non-Radioactive Cell Proliferation Assay reagent (Promega, Madison, WI). Inhibitor $\mathrm{IC}_{50}$ values were determined by plotting the curve of percentage cytopathic effect versus concentrations of NA inhibitor using Graph Pad Prism 4. The $\mathrm{CC}_{50}$ values measured the toxicity of NA inhibitors to MDCK cells and were determined by methods similar to the $\mathrm{IC}_{50}$ determination but without virus infection.

\section{Results}

\subsection{Requirement of neuraminidase activity for the formation of functional pseudotyped virus particles}

As reported in the literature, exogenous neuraminidases such as bacterial neuraminidase are required during the production of HA-pseudotyped virus (HA-PV) (Temperton et al., 2007). To study this requirement and the consequence of the neuraminidase supplementation to producer cells, influenza neuraminidase was provided by transfection of the neuraminidase expressing plasmid (from A/WSN/1933(H1N1)), along with other plasmid DNAs that expressed the influenza-HA, MuLV-GagPol, and RNA for the reporter firefly luciferase for HA-PV productions. Pseudotyped virus

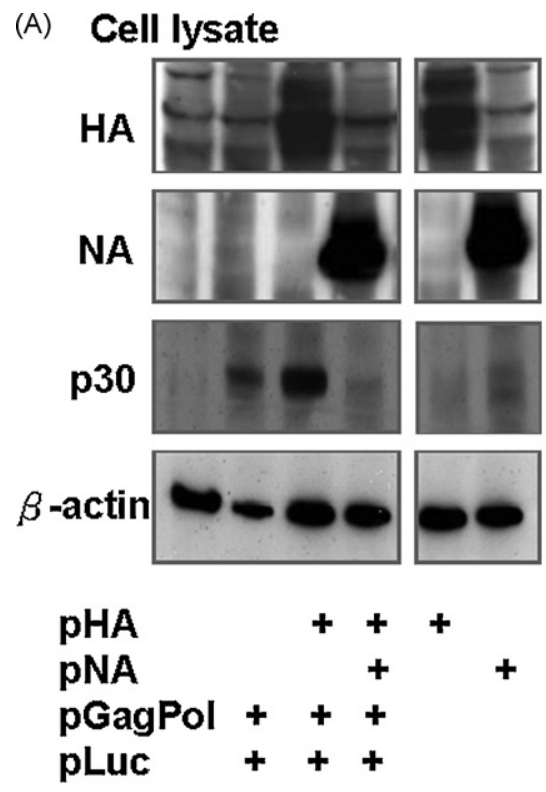

(B) Virion lysate

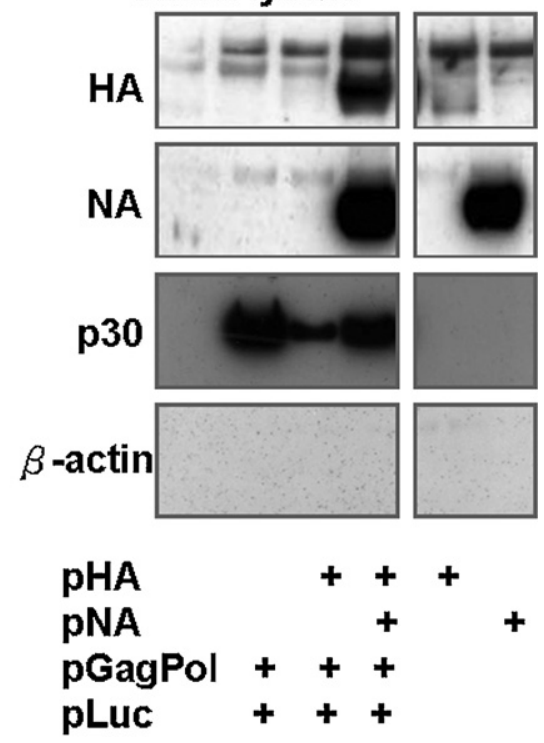

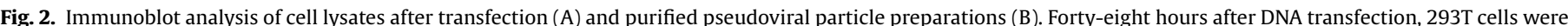

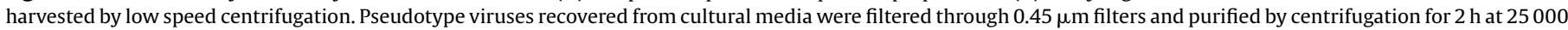

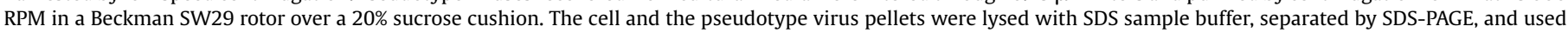

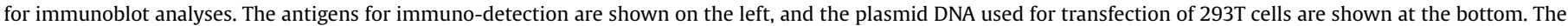

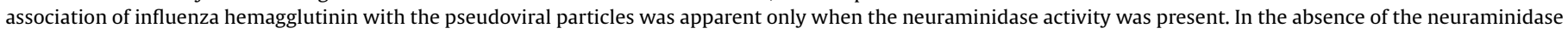

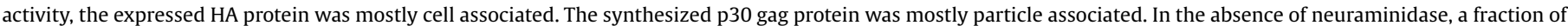
the synthesized p30 appeared to be associated with hemagglutinin and became cell-associated. 
(A) Cell lysate Virion lysate
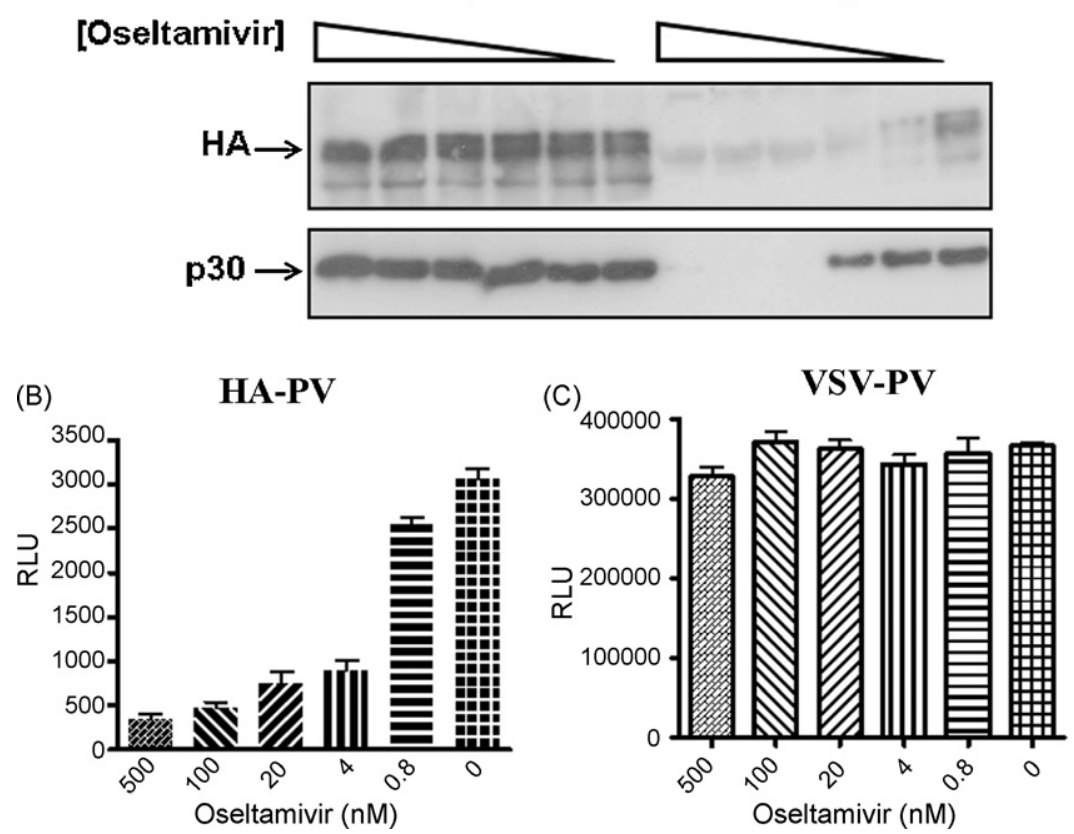

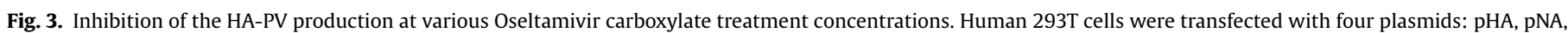

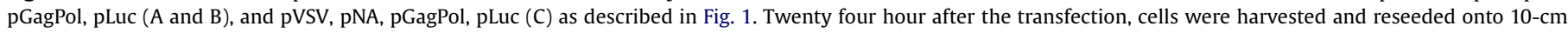

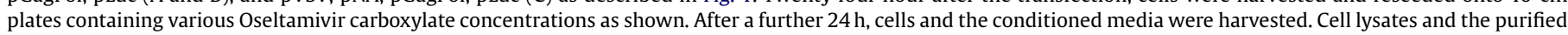

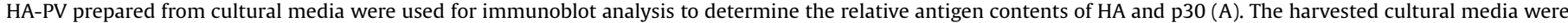
used to transduce A549 cells. The expression of firefly luciferase activities was measured 1 day after transductions (B and C).

preparations were harvested at $48 \mathrm{~h}$ after transfection and filtered through $0.45 \mu \mathrm{m}$ filters. Fig. $1 \mathrm{~A}$ shows that significant HA levels were detected only in filtrates harvested after transfection using all four plasmids. In the absence of the plasmid for NA expression, only background levels of HA were detected in the HA-PV preparations. Subsequent transduction studies also showed that functional HA-PV preparations were produced only in the presence of neuraminidase expressions (Fig. 1B). The pseudoviral particles were purified from the conditioned media of 293T cell cultures after DNA transfection. The association of influenza hemagglutinin with the pseudoviral particles was apparent only when the neuraminidase activity was provided. In the absence of the neuraminidase activity, the expressed HA protein was mostly cell associated, and substantial quantities of retroviral gag protein also became cell-associated (Fig. 2). The effect of neuraminidase expression on the HA distribution and the formation of functional pseudoviral virus preparations confirmed the neuraminidase requirement to be released from the HA-pseudotyped virus particles. This requirement also suggests that the production of HA-PV is a process resembling influenza virion release and could be used as a model mimicking the influenza release process.

\subsection{Correlation of NAI activity to the release of pseudotyped viruses}

The requirement of neuraminidase function for HA-PV production suggests that the production of HA-PV could be used for the evaluation of NAI activities. To assess this possibility, we trypsinized the 293T cells 1 day after transfection with the four plasmid DNAs and then treated the reseeded cells with various concentrations of Oseltamivir carboxylate for $24 \mathrm{~h}$ before harvesting to measure the HA-antigen levels and the functions of the pseudotype virus preparations. Fig. $3 \mathrm{~A}$ shows that the Oseltamivir carboxylate treatment appeared to inhibit the production of the HA-PV. In addition, the ability of the resulting HA-PV preparation to transduce A549 cells was also reduced, and inversely related to the Oseltamivir carboxylate concentrations used (Fig. 3B). As a control, VSV glycoprotein pseudotyped MuLV were prepared at varying Oseltamivir carboxylate concentrations, and the ability of the resulting VSVPV preparations to transduce cells were determined. The result in Fig. $3 C$ shows that unlike the production of HA-PV, the production of functional VSV-PV was unaffected by the Oseltamivir carboxylate exposures. These results thus confirm that the neuraminidase requirement is specific for HA-PV productions. Furthermore, the results also suggested the potential of using the pseudotype viral release as a cell-based assay to measure NAI potencies.

\subsection{Measurement of pseudotyped virus titers using the expressed luciferase reporter activities after transduction of recipient cells}

Our results thus far indicate that the production of HA-PV has features similar to the release of virion particles in the life cycle of influenza viruses. The HA-PV carries the RNA of firefly luciferase that could be used as a convenient reporter to titer the pseudovirus yields by transduction of recipient cells. To demonstrate that the expressed luciferase activities after transduction can be used as a measurement of HA-PV titers, HA-PV preparations were used to measure the HA antigen contents and the expressed luciferase activity levels after transduction to A549 cells. Fig. 4 shows that the measurements of the luciferase reporter activities are proportional to the pseudovirus associated HA contents determined by ELISA measurements. We thus used the measurement of the luciferase activity as a convenient quantification of the relative titers of HA-PV preparations.

\subsection{Characterization of NAIs using the pseudotype virus release assay}

HA-PV release assays were conducted to evaluate the NAI activities in 293T cells. Four NAIs, Oseltamivir carboxylate, Zanamivir, 


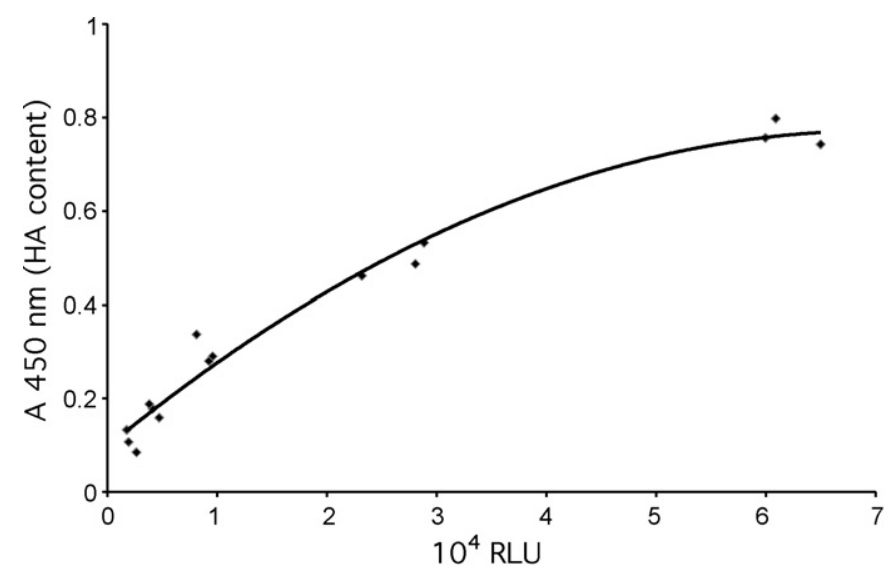

Fig. 4. Correlation of the HA-PV titers and the luciferase activities expressed upon transduction of A549 cells. HA-PV preparations at various dilutions were used to determine the relative pseudovirus titers by ELISA measurements of the HA contents. The HA-PV preparations were also used to transduce A549 cells to determine the luciferase expression levels of A549 cells at $48 \mathrm{~h}$ after transduction.

and two of Oseltamivir's phosphonate congeners (Fig. 5A) were used for this study. The HA-PV release inhibition by each of the four compounds showed a dose-dependent response, and the data were plotted to obtain $\mathrm{IC}_{50}$ values. For comparison, we expressed the same neuraminidase protein and used the recombinant enzyme to determine the $\mathrm{IC}_{50}$ values of each of the four compounds. Similar $\mathrm{IC}_{50}$ values were found for each compound determined either by the release assay or by the enzymatic assay (Fig. 5 and Table 1 ).
Table 1

$\mathrm{IC}_{50}$ values of four NAIs determined by pseudoviral release assay, neuraminidase assay, and infectivity assay against H1N1 (WSN) influenza

\begin{tabular}{llccl}
\hline Compound & NA & $\begin{array}{l}\text { Release } \\
\text { assay (nM) }\end{array}$ & $\begin{array}{l}\text { Enzyme } \\
\text { assay (nM) }\end{array}$ & $\begin{array}{l}\text { Infectivity } \\
(\mathrm{nM})\end{array}$ \\
\hline Osletamivir carboxylate & Wild type & 3.5 & 2.8 & 23 \\
Osletamivir carboxylate & H274Y & 260 & 290 & ND \\
Zanamivir & Wild type & 7.0 & 4.6 & 34 \\
Zanamivir & H274Y & 13 & 12 & ND \\
Compound 3 & Wild type & 1.6 & 1.0 & $4.3-9.5$ \\
Compound 3 & H274Y & 150 & 530 & ND \\
Compound 4 & Wild type & 0.62 & 0.10 & $0.06-0.19$ \\
Compound 4 & H274Y & 8.1 & 7.4 & ND \\
\hline
\end{tabular}

In addition, measurements were made to determine the activities of these compounds in preventing the infection of the H1N1 (WSN) influenza that has the same neuraminidase gene as the HA-PV used in the release assay. The potency rank of these compounds in the prevention of pseudoviral release parallels that of infectivity prevention (Table 1 ). Thus, although the $\mathrm{IC}_{50}$ values were different in release-prevention and anti-infection assays, the release assay can serve as guidance for antiviral potency of NAIs.

One apparent advantage of the pseudoviral release assay is the flexibility to change the pseudoviral components of HA and NA by plasmid replacements. To demonstrate this advantage, we replaced the pNA that codes for the wild type neuraminidase of H1N1 (WSN) with the plasmid pNA_H274Y carrying a site-directed mutant NA gene with tyrosine to replace histidine at position 274 (N2 nomenclature). Release inhibitions of the pseudovirus carrying H274Y
(A)<smiles>CCC(CC)O[C@H](N)C1CC(C(=O)O)=CC[C@H]1N</smiles>

1

Oseltamivir carboxylate

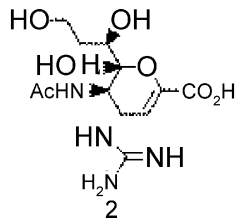

Zanamivir
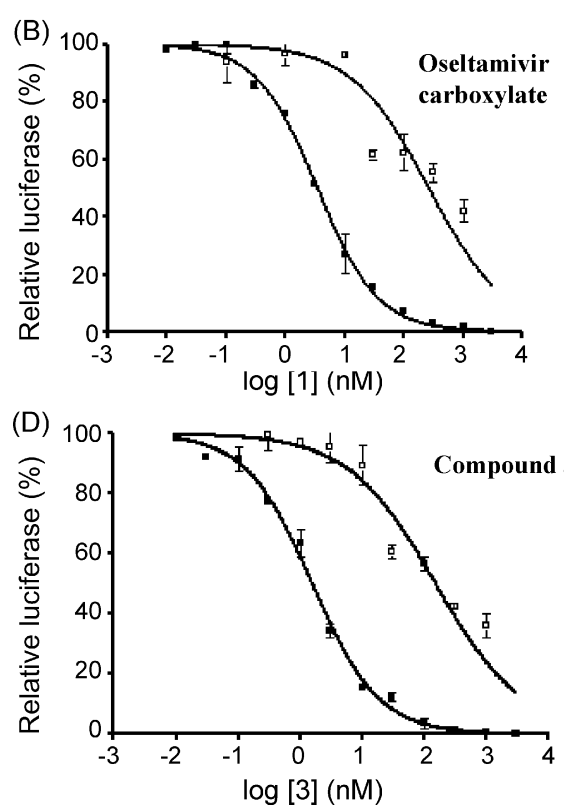<smiles>CCC(CC)O[C@H]1CC(P(=O)(O)O)=C[C@@H](N)[C@@H]1N</smiles><smiles>CCC(CC)O[C@H]1C=C(P(=O)(O)O)C[C@@H](NC(=N)N)[C@H]1NC(N)=O</smiles>
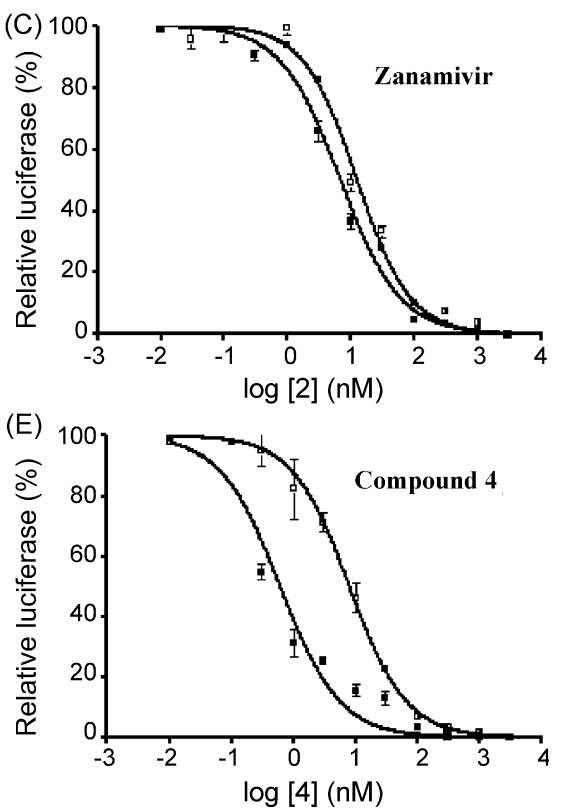

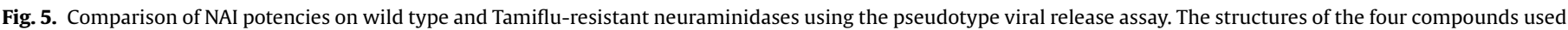

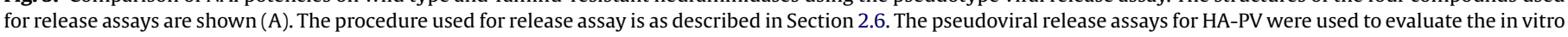

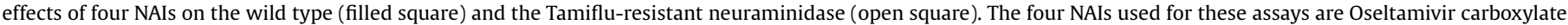
(B), Zanamivir (C), and two phosphonate analogs of Oseltamivir carboxylate: compound 3 (D) and compound 4 (E). 
neuraminidase were evaluated using the same four NAIs. Very different $\mathrm{IC}_{50}$ values were produced in this study (Fig. 5 and Table 1). Oseltamivir carboxylate and its phosphonate congeners that are less active to neuraminidase with the H274Y mutation (Shie et al., 2007) were 50-100 times less potent in the release prevention assay on pseudotype virus with H274Y neuraminidase than the wild type counterpart. In contrast, the neuraminidase inhibition by Zanamivir is somewhat insensitive to the H274Y mutation and has $\mathrm{IC}_{50}$ values similar to the wild type neuraminidase in both enzymatic and release assays. The susceptibility difference of the H274Y mutant to Oseltamivir carboxylate versus Zanamivir found in the release assays is consistent with the antiviral results reported previously (Abed et al., 2004).

\section{Discussion}

The NAIs that inhibit the influenza neuraminidase at the virus release step are used as anti-influenza drugs, and additional new anti-influenza inhibitors targeting the release step may be discovered and considered for development. The influenza release process involves the interaction of viral HA, NA, cellular lipid raft, and the sialic acid receptors (Barman and Nayak, 2007; Mishin et al., 2005; Palese et al., 1974). A model system that mimics influenza release would be useful for the understanding of the cellular and viral factors involved in the release process. This assay probably also helps confirm the inhibition mechanism of new NAI candidates and their potencies under physiological conditions. Virus-like particles and pseudotyped viruses relevant to influenza research have been described (Galarza et al., 2005; Kong et al., 2006; Lin and Cannon, 2002; Liu et al., 2005), but, to our knowledge, they have not been used to study influenza release or to explain the NAI inhibition process.

The pseudoviral release assay described in this report is specific for the NA-mediated release. It is also the first cell-based assay to measure the neuraminidase activity under physiological conditions. We used the pseudoviral release assay to evaluate the potencies of several NAIs and produced $\mathrm{IC}_{50}$ values comparable to those obtained by enzymatic assays using MUNANA. In addition, consistency in potency ranks of several NAIs were obtained in enzymatic, pseudovirus release, and infectivity assays against both wild type and drug resistant neuraminidases. The pseudoviral release assay is a slower assay in comparison to most enzymatic assays, and it is thus not a HTS assay for NAI lead discoveries. It is probably a useful assay for the evaluation of new NAIs that may have molecular interactions with factors other than neuraminidase. This consideration may be necessary particularly for the studies on influenza NAIs because both hemagglutinin and neuraminidase interact with sialic acids. Furthermore, in the viral and cellular surface, there are many different sialyl oligosaccharides that interact with both proteins with different affinities. A biochemical assay using a synthetic substrate may not be able to assess the activity of a new NAI if the physiological substrates are very different from the synthetic substrate used. In a hypothetic case, it is conceivable that the $\mathrm{IC}_{50}$ values obtained by enzymatic and infectivity assays may be very different for dual-function inhibitors that inhibit neuraminidase and also bind hemagglutinin. Pseudoviral release assays could be useful to provide explanations for these inhibitors. Such inhibitors were reported in the literature (Guo et al., 2002), although we did not have an opportunity to test them in this study using the release assay.

The pseudotype virus release assay is a versatile system, allowing the study of different NA genes or mutants by simple replacements with relevant NA expression plasmids. We showed in this report the study of NAI activities against the wild type and the Tamiflu resistant neuraminidases by simple replacement of rel- evant plasmid DNAs for experiments. The procedure for this assay is simple, and all operative procedures could be done under biosafety level two conditions. We also studied the NAI activities on the release of a different $\mathrm{HA}-\mathrm{PV}$ where the neuraminidase gene was derived from A/Hanoi/30408/2005 (H5N1). As expected, higher $\mathrm{IC}_{50}$ values for the release assay were obtained (data not shown). The alternative cell-based assay would likely to be an infectivity assay that will require higher biosafety containments.

Assessment of drug resistant variants is a practical exercise in the evaluation of new antiviral candidates. This practice may have safety concerns in the genetic manipulation of avian H5N1 viruses. The HA-pseudotyped virus being noninfectious has no safety considerations regarding genetic manipulations. Thus, it may be useful to generate a panel of HA-PVs carrying different NAI resistant mutations to screen new NAI leads for development candidates most likely to be effective against all influenza neuraminidases under physiological conditions.

In summary, the pseudovirus release assay described in this report is the first cell-based assay system for the evaluation of NAIs under conditions mimicking the physiological situations. In addition to serve as a confirmative assay, this cell-based assay, which dissociates the release process from most other infection events, may be useful in mechanism studies for inhibitors that have more complex molecular interactions.

\section{Acknowledgements}

We thank Drs. Hui-Ming Yu and Ying-Ta Wu for help preparing rabbit antibody for influenza HA and Genomics Research Center for support. We thank the National Institute of Hygiene and Epidemiology (NIHE) in Hanoi for the A/VietNam/1194/2004 HA gene. Nigel Temperton is funded by the Medical Research Council of Great Britain.

\section{References}

Abed, Y., Goyette, N., Boivin, G., 2004. A reverse genetics study of resistance to neuraminidase inhibitors in an influenza A/H1N1 virus. Antivir. Ther. 9, 577-581.

Alymova, I.V., Taylor, G., Portner, A., 2005. Neuraminidase inhibitors as antiviral agents. Curr. Drug Targets Infect. Disord. 5, 401-409.

Bantia, S., Ghate, A.A., Ananth, S.L., Babu, Y.S., Air, G.M., Walsh, G.M., 1998. Generation and characterization of a mutant of influenza $A$ virus selected with the neuraminidase inhibitor BCX-140. Antimicrob. Agents Chemother. 42, 801-807.

Barman, S., Nayak, D.P., 2007. Lipid raft disruption by cholesterol depletion enhances influenza A virus budding from MDCK cells. J. Virol. 81, 12169-12178.

Bhatia, A., Kast, R.E., 2007. How influenza's neuraminidase promotes virulence and creates localized lung mucosa immunodeficiency. Cell. Mol. Biol. Lett. 12, 111-119.

Buxton, R.C., Edwards, B., Juo, R.R., Voyta, J.C., Tisdale, M., Bethell, R.C., 2000. Development of a sensitive chemiluminescent neuraminidase assay for the determination of influenza virus susceptibility to zanamivir. Anal. Biochem. 280, 291-300.

Cabezas, J.A., Reglero, A., Hannoun, C., 1983. A fluorometric procedure for measuring the neuraminidase activity: its application to the determination of this activity in influenza and parainfluenza viruses. Anal. Biochem. 131, 121-126.

Cheam, A.L., Barr, I.G., Hampson, A.W., Mosse, J., Hurt, A.C., 2004. In vitro generation and characterisation of an influenza $B$ variant with reduced sensitivity to neuraminidase inhibitors. Antivir. Res. 63, 177-181.

Chowell, G., Miller, M.A., Viboud, C., 2007. Seasonal influenza in the United States, France, and Australia: transmission and prospects for control. Epidemiol. Infect. $1-13$.

De Clercq, E., 2006. Antiviral agents active against influenza A viruses. Nature Rev. Drug Discov. 5, 1015-1025.

França de Barros Jr., J., Sales Alviano, D., da Silva, M.H., Dutra Wigg, M., Sales Alviano, C., Schauer, R., dos Santos Silva Couceiro, J.N., 2003. Characterization of sialidase from an influenza A (H3N2) virus strain: kinetic parameters and substrate specificity. Intervirology 46, 199-206.

Galarza, J.M., Latham, T., Cupo, A., 2005. Virus-like particle (VLP) vaccine conferred complete protection against a lethal influenza virus challenge. Viral Immunol. $18,244-251$.

Gani, R., Hughes, H., Fleming, D., Griffin, T., Medlock, J., Leach, S., 2005. Potential impact of antiviral drug use during influenza pandemic. Emerg. Infect. Dis. 11, 1355-1362. 
Guo, C.T., Sun, X.L., Kanie, O., Shortridge, K.F., Suzuki, T., Miyamoto, D., Hidari, K.I, Wong, C.H., Suzuki, Y., 2002. An $\mathrm{O}$-glycoside of sialic acid derivative that inhibits both hemagglutinin and sialidase activities of influenza viruses. Glycobiology $12,183-190$

Hampson, A.W., 2006. Avian influenza: a pandemic waiting in the wings? Emerg. Med. Aust. 18, 420-429.

Hurt, A.C., Selleck, P., Komadina, N., Shaw, R., Brown, L., Barr, I.G., 2007. Susceptibility of highly pathogenic $\mathrm{A}(\mathrm{H} 5 \mathrm{~N} 1)$ avian influenza viruses to the neuraminidase inhibitors and adamantanes. Antivir. Res. 73, 228-231.

Katinger, D., Mochalova, L., Chinarev, A., Bovin, N., Romanova, J., 2004. Specificity of neuraminidase activity from influenza viruses isolated in different hosts tested with novel substrates. Arch. Virol. 149, 2131-2140.

Kiyotani, K., Takei, N., Senoo, M., Takao, S., Otsuki, K., Tsubokura, M., Yoshida, T. 1987. Enzymological characteristics of avian influenza A virus neuraminidase. Microbiol. Immunol. 31, 1131-1135.

Kobasa, D., Kodihalli, S., Luo, M., Castrucci, M.R., Donatelli, I., Suzuki, Y., Suzuki, T., Kawaoka, Y., 1999. Amino acid residues contributing to the substrate specificity of the influenza A virus neuraminidase. J. Virol. 73, 6743-6751.

Kong, W.P., Hood, C., Yang, Z.Y., Wei, C.J., Xu, L., Garcia-Sastre, A., Tumpey, T.M., Nabel, G.J., 2006. Protective immunity to lethal challenge of the 1918 pandemic influenza virus by vaccination. Proc. Natl. Acad. Sci. U.S.A. 103, 1598715991.

Li, K.S., Guan, Y., Wang, J., Smith, G.J., Xu, K.M., Duan, L., Rahardjo, A.P., Puthavathana, P., Buranathai, C., Nguyen, T.D., Estoepangestie, A.T., Chaisingh, A. Auewarakul, P., Long, H.T., Hanh, N.T., Webby, R.J., Poon, L.L., Chen, H., Shortridge, K.F., Yuen, K.Y., Webster, R.G., Peiris, J.S., 2004. Genesis of a highly pathogenic and potentially pandemic H5N1 influenza virus in eastern Asia. Nature 430, 209213.

Lin, A.H., Cannon, P.M., 2002. Use of pseudotyped retroviral vectors to analyze the receptor-binding pocket of hemagglutinin from a pathogenic avian influenza $A$ virus (H7 subtype). Virus Res. 83, 43-56.
Liu, H.L., Rong, L.J., Zhou, B., Wei, J.C., Zheng, Q.S., Chen, P.Y., 2005. Characterization of murine leukemia virus recombinants that express H5N1 subtype avian influenza virus hemagglutinin glycoproteins. Sheng Wu Gong Cheng Xue Bao 21, 47-51.

Mishin, V.P., Novikov, D., Hayden, F.G., Gubareva, L.V., 2005. Effect of hemagglutinin glycosylation on influenza virus susceptibility to neuraminidase inhibitors. J. Virol. 79, 12416-12424.

Molla, A., Kati, W., Carrick, R., Steffy, K., Shi, Y., Montgomery, D., Gusick, N., Stoll, V.S., Stewart, K.D., Ng, T.I., Maring, C., Kempf, D.J., Kohlbrenner, W., 2002. In vitro selection and characterization of influenza $A(A / N 9)$ virus variants resistant to a novel neuraminidase inhibitor A-315675. J. Virol. 76, 5380-5386.

Onodera, S., 1994. A microplate assay for sialidase activity using plant lectin binding to $\mathrm{N}$-acetyllactosamine. Biol. Pharm. Bull. 17, 29-33.

Palese, P., Tobita, K., Ueda, M., Compans, R.W., 1974. Characterization of temperature sensitive influenza virus mutants defective in neuraminidase. Virology 61, 397-410.

Poland, G.A., Jacobson, R.M., Targonski, P.V., 2007. Avian and pandemic influenza: an overview. Vaccine 25, 3057-3061.

Sato, K., Hanagata, G., Kiso, M., Hasegawa, A., Suzuki, Y., 1998. Specificity of the N1 and N2 sialidase subtypes of human influenza A virus for natural and synthetic gangliosides. Glycobiology 8, 527-532.

Shie, J.J., Fang, J.M., Wang, S.Y., Tsai, K.C., Cheng, Y.S., Yang, A.S., Hsiao, S.C., Su, C.Y., Wong, C.H., 2007. Synthesis of tamiflu and its phosphonate congeners possessing potent anti-influenza activity. J. Am. Chem. Soc. 129, 11892-11893.

Temperton, N.J., Hoschler, K., Major, D., Nicolson, C., Manvell, R., Hien, V.M., Ha, D.Q., de Jong, M., Zambon, M., Takeuchi, Y., Weiss, R.A., 2007. A sensitive retroviral pseudotype assay for influenza H5N1-neutralizing antibodies. Influenz. Other Respir. Vir. 1, 105-112.

Vila-Córcoles, A., Rodriguez, T., de Diego, C., Ochoa, O., Valdivieso, A., Salsench, E., Ansa, X., Badia, W., Saún, N., 2007. Effect of influenza vaccine status on winter mortality in Spanish community-dwelling elderly people during 2002-2005 influenza periods. Vaccine 25, 6699-6707. 